\title{
Schlechtes Vorbild
}

\author{
Wenig Resonanz für Grippeimpfung bei Krankenhauspersonal
}

Medizinisches Personal in Kliniken ist eine mögliche Infektionsquelle für Patienten, die oft wegen Grunderkrankungen ein erhöhtes Risiko für schwere und eventuell sogar tödliche Grippe-Verläufe haben. Doch ausgerechnet Krankenhausmitarbeiter lassen sich zum größten Teil nicht impfen. Laut Robert Koch Institut (RKI) geben Ärzte dafür andere Gründe als Pfleger an.

Die Ständige Impfkommission (STIKO) betont daher in ihren aktuellen Informationen zum Grippeschutz: „Die Impfung des Personals folgt dem ethischen Gebot, Patienten nicht zu schaden. Gleichzeitig dient sie dem persönlichen Schutz bei beruflichem Patientenkontakt." Trotz dieses dringenden Appells lassen sich die meisten Mitarbeiter in Kliniken nicht impfen, wie eine Online-Befragung des Personals zweier Universitätskliniken bestätigte (1). Von den etwa 8500 per E-Mail kontaktierten Personen antwortete etwa jeder fünfte. Gefragt wurde nach dem Impfschutz in der aktuellen Influenza-Saison und nach Einstellungen der Mitarbeiter zur GrippeImpfung.

Nur etwa 56 Prozent des ärztlichen Personals und nur knapp 35 Prozent des Pflegepersonals hatten den Grippeschutz. Mitarbeiter in der Pflege mit häufigem Patientenkontakt waren dabei sogar seltener geimpft als Personal mit gelegentlichem Kontakt zu Kranken wie in der Verwaltung (36 \% Impfrate), Technik (44 \%) oder im
Labor (45\%). Je jünger die Mitarbeiter waren, desto geringer waren die Impfraten: Bei den 18- bis 24-Jährigen betrug sie 25 Prozent, bei den 45 - bis 54-Jährigen 48 Prozent.

\section{Viele Vorurteile gegenüber Grippeimpfung}

Befragt nach Gründen für den fehlenden Schutz ergaben sich viele falsche Vorstellungen: Fast jeder Fünfte gab an, wegen eines guten Immunsystems ein geringes Influenza-Risiko zu haben. Ebenso viele befürchteten, dass die Impfung selbst eine Grippe auslösen könne. Jeder Sechste hält eine Influenza für nicht gefährlich. Immerhin jeder Zehnte hält die Impfung für unwirksam und jeder Zwanzigste gab an, generell gegen Impfungen zu sein. Die Gründe, sich nicht impfen zu lassen, unterschieden sich dabei zwischen den Berufsgruppen: Bei den Ärzten spielten vor allem organisatorische Gründe eine maßgebliche Rolle wie "habe es vorgehabt, aber nicht geschafft“ oder „, habe es vergessen“. Etwa 67 Prozent der Ärzte, aber nur 44 Prozent des medizinischen Personals wünschen sich ein Impfangebot vor Ort in der Klinik.

\section{Pflegepersonen befürchten häufiger Nebenwirkungen}

Beim Personal in der Pflege wurde auch häufig Angst vor Nebenwirkungen als

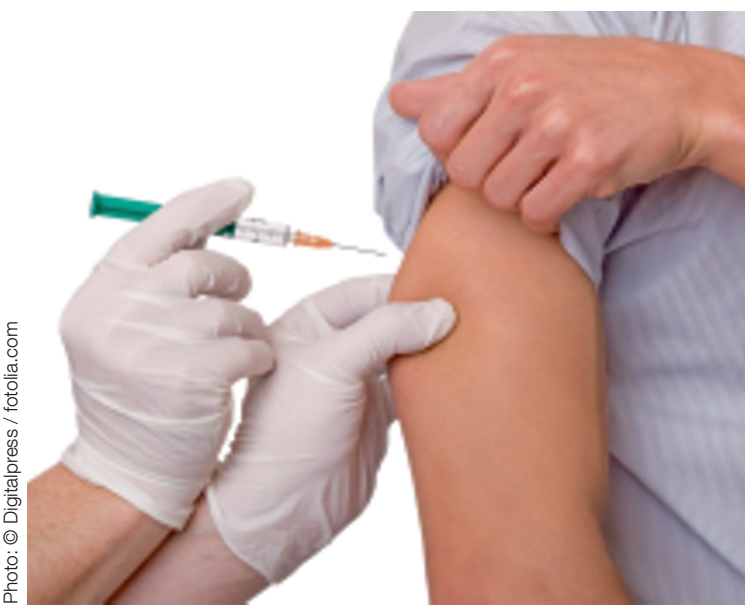

Die Grippeimpfung des Krankenhauspersonals schützt auch die Patienten.

Grund für den fehlenden Impfschutz genannt. Das RKI plant, die Umfrage zu den Impfquoten auch auf andere Krankenhäuser auszudehnen. Die Ergebnisse könnten Maßnahmen zur Verbesserung der Impfraten unterstützen. Voraussetzung für robuste Daten sei allerdings eine breite Unterstützung der teilnehmenden Kliniken, so das RKI.

$\ddot{A} Z / w . g$.

\section{LITERATUR}

1. Epi Bull 2016; 47: 521

\footnotetext{
IMPRESSUM

Herausgeber und Verleger: Springer-Verlag GmbH, Prinz-Eugen-Str. 8-10, Postfach 11, 1040 Wien, Austria, Tel.: $01 / 33024$ 15, Fax: $01 / 3302426$ Internet: www.springer.com, www.SpringerMedizin.at Geschäftsführung: Joachim Krieger, Dr. Alois Sillaber, Dr. Heinrich Weinheimer Leitung Journale und Redaktionen Medizin: Gabriele Hollinek Redaktion: Verantwortlicher Redakteur: Verena Kienast, Dipl. Tzt. Elise Haidenthaller Leitung Verkauf Medizin: Robert Seiwald Anzeigen: Gabriele Popernitsch Mediaservice: Claudia Trischler; Es gilt die Anzeigenpreisliste 2016. Erscheinungsweise: 10x jährlich Abonnement: Michaela Bolli; Bezugspreis pro Jahr: für Institutionen EUR 130,90 zuzüglich MwSt. und Versandkosten Bezugsbedingungen: Das Abonnement für Einzelbezieher gilt mit Bezug des ersten Heftes jeweils für ein Jahr mit der in der Preisliste für einen vollen Jahrgang angegebenen Anzahl von Ausgaben. Abbestellungen innerhalb dieser Laufzeit können nicht entgegengenommen werden. Das Abonnement der Zeitschrift verlängert sich automatisch um ein weiteres Jahr, wenn nicht bis 2 Monate vor Ablauf des Abonnements beim Verlag eine schriftliche Kundigung eingegangen ist. Verlagsort: Wien Herstellungsort: Linz Erscheinungsort: Wien Verlagspostamt: 1040 Wien P.b.b. ISSN Print: 0949-7323, ISSN Electronic: 1613-7574, Band 21, Heft 10/2016 Layout: KM-Satz Design: Wojtek Grzymala Druck: Friedrich Druck \& Medien GmbH, Linz, Austria Wissenschaftlicher Beirat: FH-Prof. Dr. Holger Penz, Feldkirchen; Univ.-Prof. Dr. Christa Lohrmann, Graz; Univ-Prof. Dr. Christa Them, Hall i. T.; Univ. Prof. Hanna Mayer, Wien; FH-Prof. Dr. Roswith Engel, Wien, Univ.-Prof. Dr. Jürgen Osterbrink, Salzburg; Dr. Elisabeth Rappold, Wien; Maria Jesse, Wien; Mag. Martina Kuttig, Krems; Charlotte Staudinger, Wien. Dr. Eleonore Kemetmüller, Krems. Alle namentlich gekennzeichneten Beiträge spiegeln nicht unbedingt die Meinung der Redaktion wider. Diese Beiträge fallen somit in den personlichen Verantwortungsbereich des Verfassers. Die Redaktion übernimmt keine Haftung für unaufgefordert eingesandte Manuskripte. Mit „Sonderbericht“ oder „Advertorial“ gekennzeichnete Seiten sind entgeltliche Einschaltungen nach \$26 Mediengesetz. Allgemeiner Teil/Rechtliche Hinweise für Autoren Die Autorin/der Autor erklärt, dass ihr/sein Manuskript in dieser Form bislang nicht anderweitig veröffentlicht ode zur Veröffentlichung eingereicht wurde. Die Autorin/der Autor überträgt mit der Übergabe des fertigen Manuskripts und der Veröffentlichung in der Fachzeitschrift die notwendigen Nutzungs-
rechte zur Vervielfältigung und Verbreitung an den Verlag, insbesondere das Recht der Nutzung zu gewerblichen Zwecken durch Druck, Nachdruck, Verbreitung in elektronischer Form oder rechte zur Vervielfältigung und Verbreitung an den Verlag, insbesondere das Recht der Nutzung zu gewerblichen Zwecken durch Druck, Nachdruck, Verbreitung in elektronischer Form oder andere Verfahren und Medien durch Springer Nature. Beiträge, die in procare erscheinen, können auch in der Springer-Zeitschrift Heilberufe veröffentlicht werden. Die Autorin/der Autor holt, falls notwendig, die Nutzungsrechte an Texten und Bildern Dritter vor Übergabe des fertigen Manuskripts ein, eventuelle Ansprüche Dritter sind somit geklärt. Hinweise zur Verwertung: Die
Zeitschrift sowie alle in ihr enthaltenen einzelnen Beiträge und Abbildungen sind urheberrechtlich geschützt. Jede Verwertung, auch auszugsweise, die nicht ausdrücklich vom UrheberrechtsZeitschrift sowie alle in ihr enthaltenen einzelnen Beiträge und Abbildungen sind urheberrechtlich geschützt. Jede Verwertung, auch auszugsweise, die nicht ausdrücklich vom Urheberrechts-
gesetz zugelassen ist, bedarf der vorherigen schriftlichen Zustimmung des Verlages. Das gilt insbesondere für Vervielfältigungen, Bearbeitungen, Übersetzungen, Mikroverfilmungen und die gesetz zugelassen ist, bedarf der vorherigen schriftlichen Zustimmung des Verlages. Das gilt insbesondere für Vervielfältigungen, Bearbeitungen, Übersetzungen, Mikroverfilmungen und die
Verarbeitung in elektronischen Systemen. Produkthaftung: Die Wiedergabe von Gebrauchsnamen, Handelsnamen, Warenbezeichnungen usw. in dieser Zeitschrift berechtigt auch ohne besonVerarbeitung in elektronischen Systemen. Produkthaftung: Die Wiedergabe von Gebrauchsnamen, Handelsnamen, Warenbezeichnungen usw. in dieser Zeitschrift berechtigt auch ohne besondere Kennzeichnung nicht zu der Annahme, dass solche Namen im Sinne der Warenzeichen- und Markenschutz-Gesetzgebung als frei zu betrachten wären und daher von jedermann benutz
werden dürften. Angaben über Dosierungsanweisungen und Applikationsformen sind anhand anderer Literaturstellen oder der Packungsbeilage auf ihre Richtigkeit zu überprüfen. Der Verlag werden durrtten. Angaben über Dosierungsanweisungen und Applikationsformen sind anhand anderer Literaturstellen oder der Packungsbeilage auf ihre Richtigkeit zu überprüfen. Der Verlag ubernimmt hierfür keine Gewähr. Eigentümer und Copyright-Inhaber: @ 2016 Springer-Verlag/Wien. Springer Medizin ist Teil von Springer Nature. Coverbild: @ MAY / BSIP / mauritius images
} 